\title{
A brief note on the approach to the conic sections of a right circular cone from dynamic geometry
}

\author{
Eugenio Roanes-Lozano
}

\begin{abstract}
Nowadays there are different powerful 3D dynamic geometry systems (DGS) such as GeoGebra 5, Calques 3D and Cabri Geometry 3D. An obvious application of this software that has been addressed by several authors is obtaining the conic sections of a right circular cone: the dynamic capabilities of 3D DGS allows to slowly vary the angle of the plane w.r.t. the axis of the cone, thus obtaining the different types of conics. In all the approaches we have found, a cone is firstly constructed and it is cut through variable planes. We propose to perform the construction the other way round: the plane is fixed (in fact it is a very convenient plane: $z=0$ ) and the cone is the moving object. This way the conic is expressed as a function of $x$ and $y$ (instead of as a function of $x, y$ and $z$ ). Moreover, if the 3D DGS has algebraic capabilities, it is possible to obtain the implicit equation of the conic.
\end{abstract}

Mathematics Subject Classification (2010). Primary 68U99; Secondary 97G40; Tertiary 68 U05.

Keywords. Conics, Dynamic Geometry, 3D Geometry, Computer Algebra.

\section{Introduction}

Nowadays there are different powerful 3D dynamic geometry systems (DGS) such as GeoGebra 5 [2], Calques 3D [7, 10] and Cabri Geometry 3D [11].

Focusing on the most widespread one (GeoGebra 5) and conics, there is a "conics" icon in the ToolBar. This tool constructs a conic:

- given five points on it, or

- given either the two foci and a point on the conic or the focus and the directrix of the conic (depending on which conic is considered).

Obviously, a conic can also be plot from its equation, if it is known.

But a more interesting first approach is the constructive one that uses the properties of the conics to draw them (such as the gardener's method to draw ellipses). The equation of the conic can be derived from certain given elements of the conic (for instance, the coordinates of the foci and the sum of distances to the foci for the ellipse). In fact, there are many papers on loci determination (in general) using DGS, some even applied to education [5]. This constructive approach can also be applied to quadrics using a 3D DGS [6].

This approach is somehow followed in university textbooks like [1], where conics are introduced in two steps: a first step, where only high school mathematics techniques are required, and a second one, requiring techniques introduced along the course, in order the students to be able to classify conics. For instance, in the Spanish educational system, conics are introduced at the end of the Secondary Education, although they are treated in detail at university in the first year of the 
bachelor's degrees programs in mathematics, physics, engineering, etc. Therefore, the approach proposed here is adequate for undergraduates, although other very general and formal approaches like [3] exist.

Let us note that the constructive approach requires of effective algebraic methods that can be performed directly by the DGS, if it has algebraic capabilities [2], or externally by a CAS [8]. Let us observe that GeoGebra 5 offers a Locus tool for the 2D case, but it is unavailable in the 3D case (although computer packages on automatic 3D loci determination do exist $[4,8]$ ).

Focusing on the specific topic of this paper, the conic sections of a right circular cone, it is an obvious application of 3D DGS that has been addressed by several authors (see, for example, [9]). The dynamic capabilities of 3D DGS allows to slowly vary the angle of the plane w.r.t. the axis of the cone, thus obtaining the different types of conics. But, in all the approaches we have found, a cone is firstly constructed and it is cut through variable planes (see Figure 1).

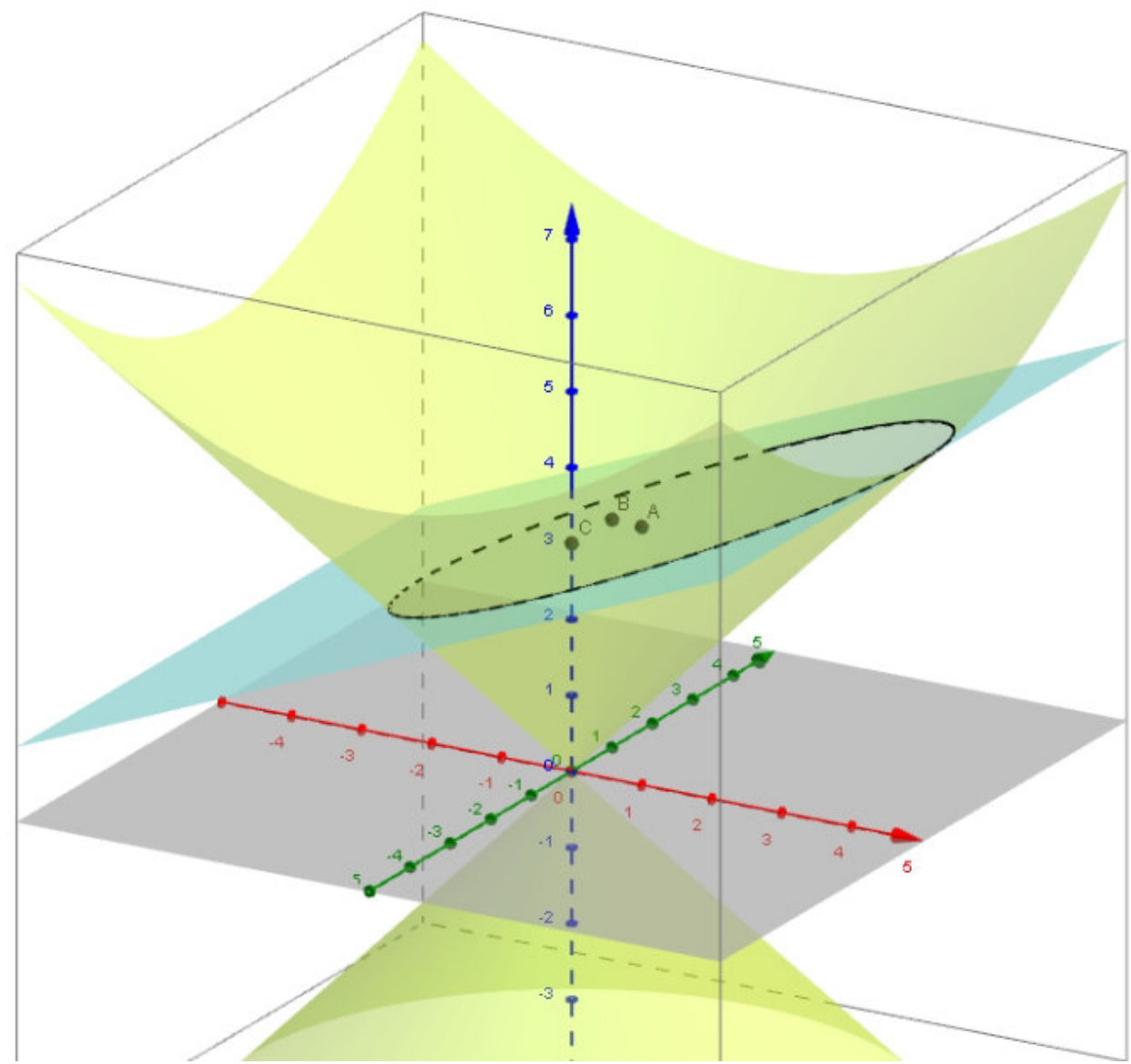

FIGURE 1. Usual approach to the conic sections of a right circular cone: the cone is fixed and the plane can be dragged and dropped.

But, if this approach is followed, a curve in the space is obtained. For instance, in the example of Figure 1, GeoGebra 5 returns the expression:

$$
X=(1.43,0,3.57)+(-3.57 \cos (t),-3.27 \sin (t),-1.43 \cos (t))
$$


A brief note on the approach to the conic sections of a right circular cone from dynamic geometry3

that is correctly classified in the so called Construction Protocol of GeoGebra 5 as an ellipse (hyperbolae and parabolae are also correctly recognized, but the degenerated case "pair of intersecting lines" isn't -it just says: "Intersection curve of $\mathrm{P}$ and $\mathrm{S}$ "). Clearly, this construction returns the vector equation of a curve (conic) in the space.

Obtaining the conics as sections of a right circular cone is due to Apollonius of Perga. The term "Apollonius cone" is often used to denote a figure or model of a cone showing its different conic sections.

\section{The approach proposed}

\subsection{Defining and conveniently allocating the geometric objects of the construction}

The proposed approach can be visualized in Figure 2. The key idea is to intersect the cone with plane $z=0$, so that the intersecting curve is expressed in variables $x$ and $y$.

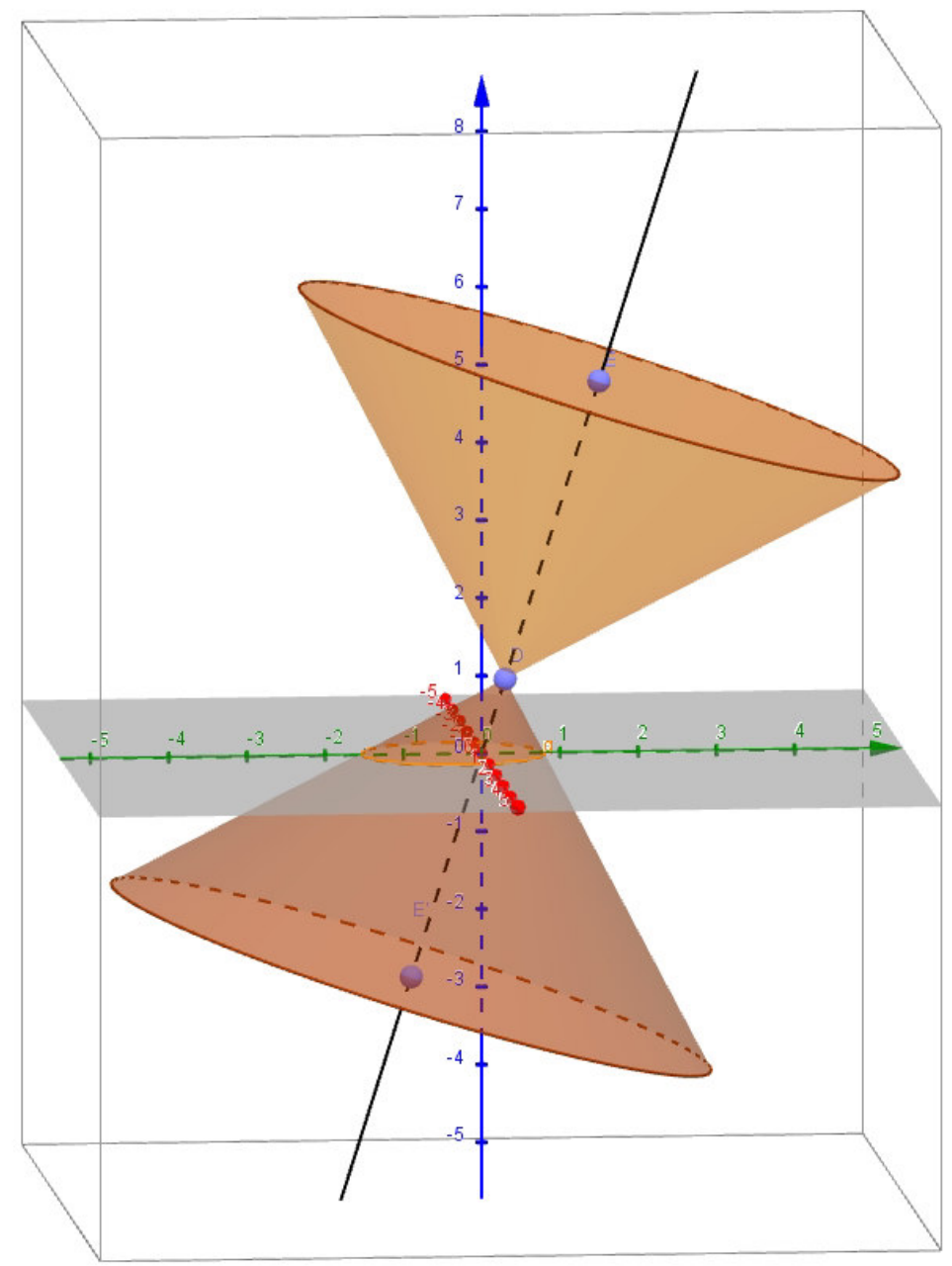

FIGURE 2. The proposed approach to the conic sections of a right circular cone: the plane $(z=0)$ is fixed and the cone can be dragged and dropped. 
We shall illustrate the construction in the DGS GeoGebra 5. The cone shouldn't change its form when dragged and dropped. We can therefore consider a cone (Figure 3) such that:

i) its apex lies on the circle contained in plane $x=0$, of centre $(0,0,0)$ and radius 1 ,

ii) its axis is a line through $(0,0,0)$ contained in plane $x=0$,

iii) its apex angle is initially set to $90^{\circ}$ (for the sake of simplicity, for instance for easily finding the parabola case, a "border case" between ellipse and hyperbola), although this can be obviously changed.

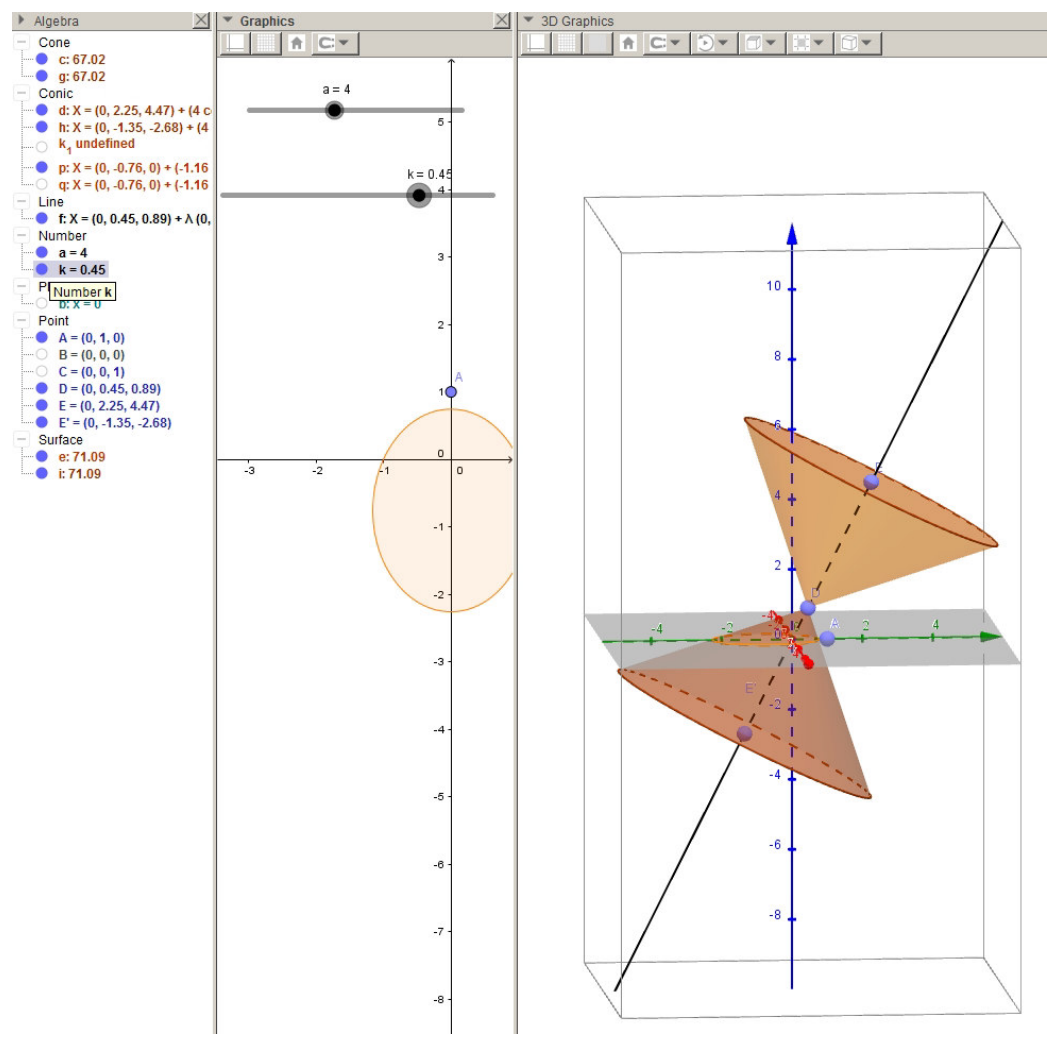

FIGURE 3. The Algebra, Graphics and Graphics $3 D$ windows of GeoGebra 5 simultaneously opened in the proposed construction.

The advantages of this construction are the following:

- as already mentioned, the cone is not altered when dragged and dropped,

- as said above, intersecting the cone with plane $z=0$ has the advantage of obtaining a curve expressed in variables $x$ and $y$,

- from i) and ii), the axis of the conics will always be the $y$ axis,

- from i) and ii), the special cases "circle" and "pair of intersecting lines" correspond to a cone with vertical and horizontal axis (respectively),

- from iii) (apex angle initially set to $90^{\circ}$ ), the parabola case, a "border case" between ellipse and hyperbola, corresponds to a cone which axis is the angle bisector of the $y$ and $z$ axis (in plane $x=0)$. 
A brief note on the approach to the conic sections of a right circular cone from dynamic geometry5

\subsection{Defining and allocating the geometric objects of the construction}

In GeoGebra 5 a cone is defined by its apex $(D)$, another point on its axis $(E)$, and the radius $(a)$ of its basis through the second point. For the shape and size not to be altered when point $D$ is dragged and dropped:

- $D$ can be defined as the point $\left(0, k, \sqrt{1-k^{2}}\right)$,

- $E$ can be defined as the image of $D$ in the homotethy of centre $(0,0,0)$ and ratio, for instance, 5 (therefore the length of $\overline{D E}$ is 4 ),

- for the apex angle to be $90^{\circ}$, the radius $a$ is assigned the value of the length of $\overline{D E}$.

Then we can ask GeoGebra 5 to intersect the cone and the plane ( $p$ is the intersection curve).

The symmetric of this cone w.r.t. point $D$ should be constructed too (Figure 3). Let us remember that we have tried to keep the construction as simple as possible, therefore using GeoGebra 5 Cone Tool (that creates a solid). Another option would be to consider the algebraic variety of the ideal generated by the algebraic equation of the cone (as done in Figure 1).

It is advisable to built sliders for $a$ (in case the user would like to change the apex angle) and $k$ (in order to easily change the slope of the axis -and consequently the intersection conic), the later with a -1 to 1 range.

\section{Obtaining the conic sections of a right circular cone}

Using the construction described, the different conic sections of a right circular cone can be easily obtained using GeoGebra 5. With the notation for variables introduced above, we have (Figure 4):

- Value of the parameter: $k=0$

Conic: Circle.

Equation: $p:=X=(0,0,0)+(\cos (t), \sin (t), 0)$

- Value of the parameter in the interval $\left(0, \frac{\sqrt{2}}{2}\right)$, for example: $k=0.55$

Conic: Ellipse.

Equation (coefficients are rounded): $p:=X=(0,-1.39,0)+(-1.33 \sin (t), 2.11 \cos (t), 0)$

- Value of the parameter: $k=\frac{\sqrt{2}}{2}$

Conic: Parabola.

Equation (coefficients are rounded): $p:=X=(0,0.71,0)+\left(-0.71 t,-0.35 t^{2}, 0\right)$

- Value of the parameter in the interval $\left(\frac{\sqrt{2}}{2}, 1\right)$, for example: $k=0.85$

Conic: Hyperbola.

Equation (coefficients are rounded): $p:=X=(0,1.91,0)+(-0.79 \sinh (t), \pm 1.18 \cosh (t), 0)$

- Value of the parameter: $k=1$

Conic: Pair of intersecting lines (GeoGebra 5 simply answers: "Intersection curve of $\mathrm{P}$ and S").

Equation: $p:=X=(0,1,0)+\lambda(1, \pm 1,0)$

(as said above, the kind of conic is specified in the Construction Protocol).

Let us underline that GeoGebra 5 returns the vector equation of the conic:

- using algebraic expressions for the parabola and pair of intersecting lines,

- using trigonometric functions for the circle and ellipse, and

- using hyperbolic functions for the hyperbola.

Regarding the plots in (Figure 4), GeoGebra 5 consider the cones as solids, not as algebraic surfaces (the cones are limited by their bases), as already mentioned. Consequently, an ugly segment appears in all cases, except for the circle and the ellipse (the spurious segment is the intersection of the basis(es) of the solid cone(s) with the plane). 


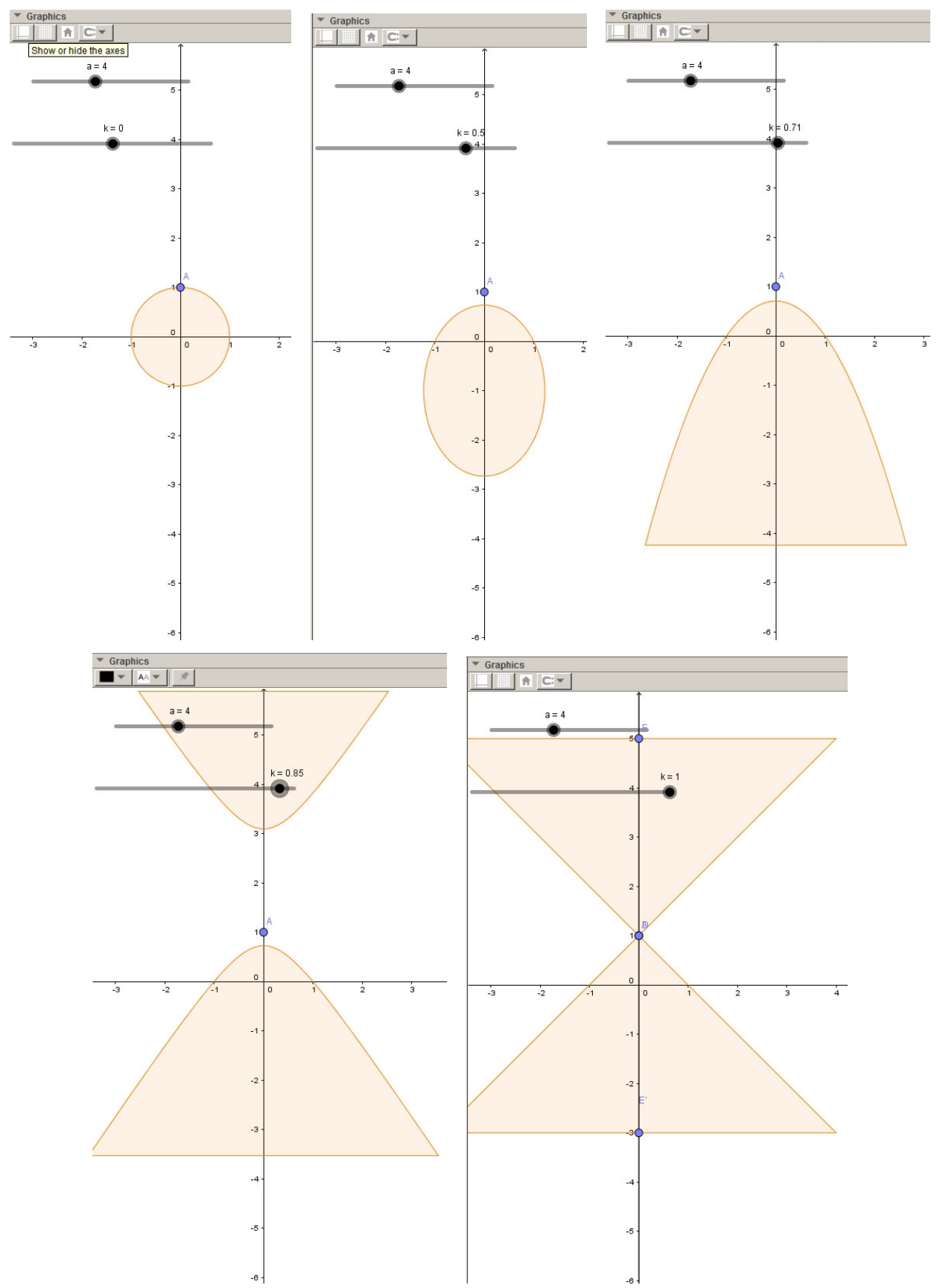

FIGURE 4. The different conic sections of a right circular cone obtained.

\section{Obtaining the implicit equation of the different conics}

With the construction in this approach, the $y$ axis is the axis of the conic. So we can perform the following process in the CAS window: 
A brief note on the approach to the conic sections of a right circular cone from dynamic geometry7

1) Execute the definition of the intersecting curve (of plane and cone). A vector equation is obtained.

2) Express the previous equation in matrix format.

3) Compute $x$ minus the result of substituting $\cos (t)$ and $\sin (t)$ (if any) by $t$ and $\sqrt{1-t^{2}}$ (respectively) in the first element of the matrix ${ }^{1}$.

4) Compute $y$ minus the result of substituting $\cos (t)$ and $\sin (t)$ (if any) by $t$ and $\sqrt{1-t^{2}}$ (respectively) in the second element of the matrix, minus the trailing coefficient ${ }^{2}$.

5) Eliminate $t$ in the algebraic system consisting in the two equations obtained in the previous two steps.

6) Split the resulting expression in two, equalize as an equation, square both sides and restore as a polynomial expression.

7) Divide by the absolute value of the trailing coefficient.

8) Approximate, if necessary.

Let us underline that the process is dynamic (if the value of $k$ is changed, all subsequent computations are updated). For example:

- An ellipse is obtained when $k=0.55$ :

$$
0.57 x^{2}+0.22 y^{2}-1
$$

(the coefficients are rounded here for the sake of brevity). The complete process and the exact equation are shown in Figure 5.

- A circle is obtained when $k=0$ :

$$
x^{2}+y^{2}-1
$$

by following the same approach (see Figure 6).

- A parabola is obtained when $k=\frac{\sqrt{2}}{2}$ :

$$
0.71 x^{2}+y
$$

(the coefficients are rounded here for the sake of brevity). The complete process and the exact equation are shown in Figure 7.

Unfortunately, when the conic is a hyperbola, $\sinh (t)$ and $\cosh (t)$ expressions arise, and the CAS of GeoGebra 5 doesn't handle the expressions in a similar way as algebraic or trigonometric expressions (at least we haven't been able to compute them).

\footnotetext{
${ }^{1}$ The reason for using the same parameter $(t)$ and not another one is that, in the parabola case, $t$ is the parameter chosen by the system. This substitution is made in order to transform the system into an algebraic one.

${ }^{2}$ Subtracting the trailing coefficient from $y$ corresponds to a reference system change (in order to simplify the equation of the conic).
} 


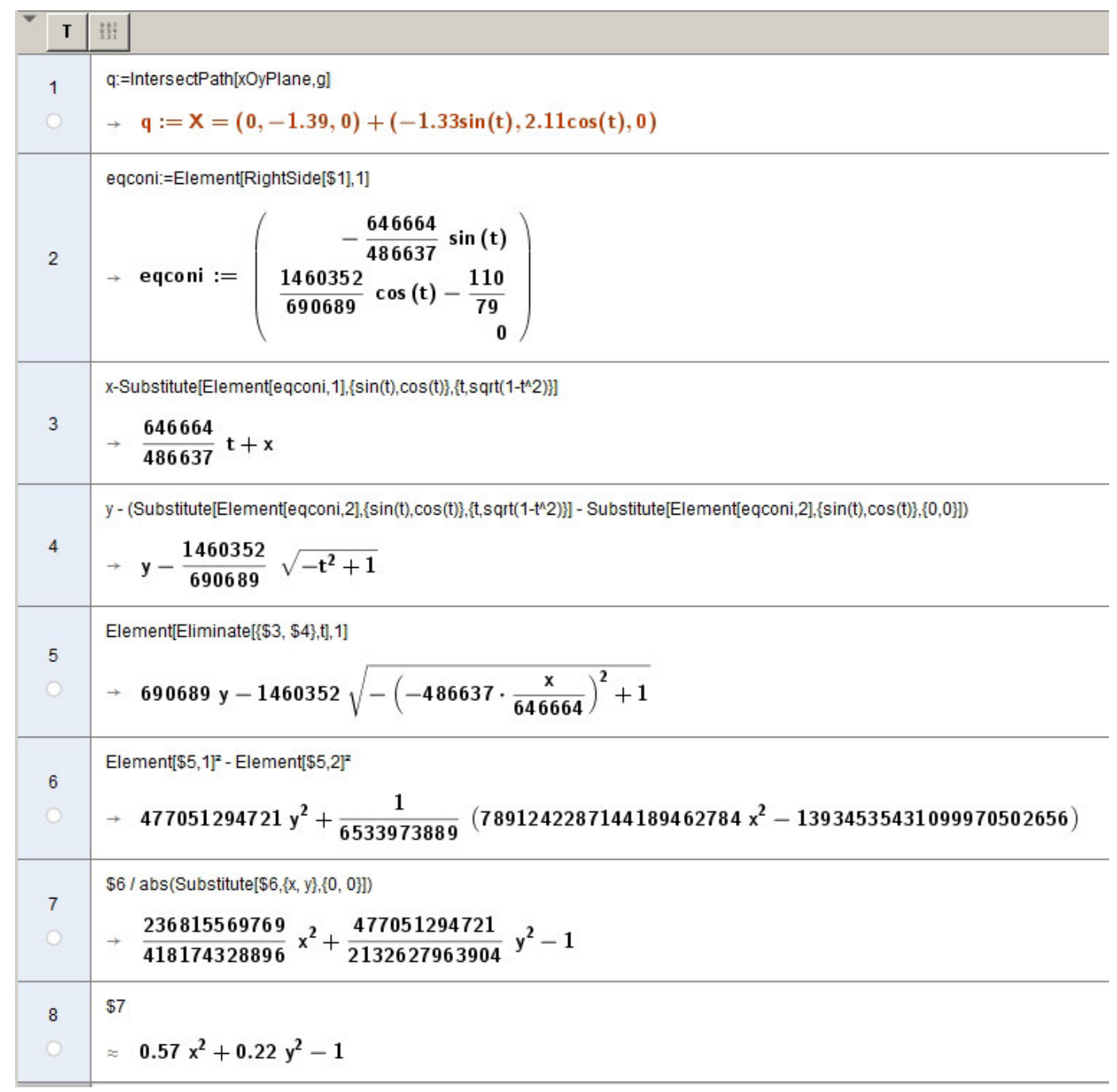

FIGURE 5. Obtaining the implicit equation of a certain ellipse as a conic section of a right circular cone. 
A brief note on the approach to the conic sections of a right circular cone from dynamic geometry9

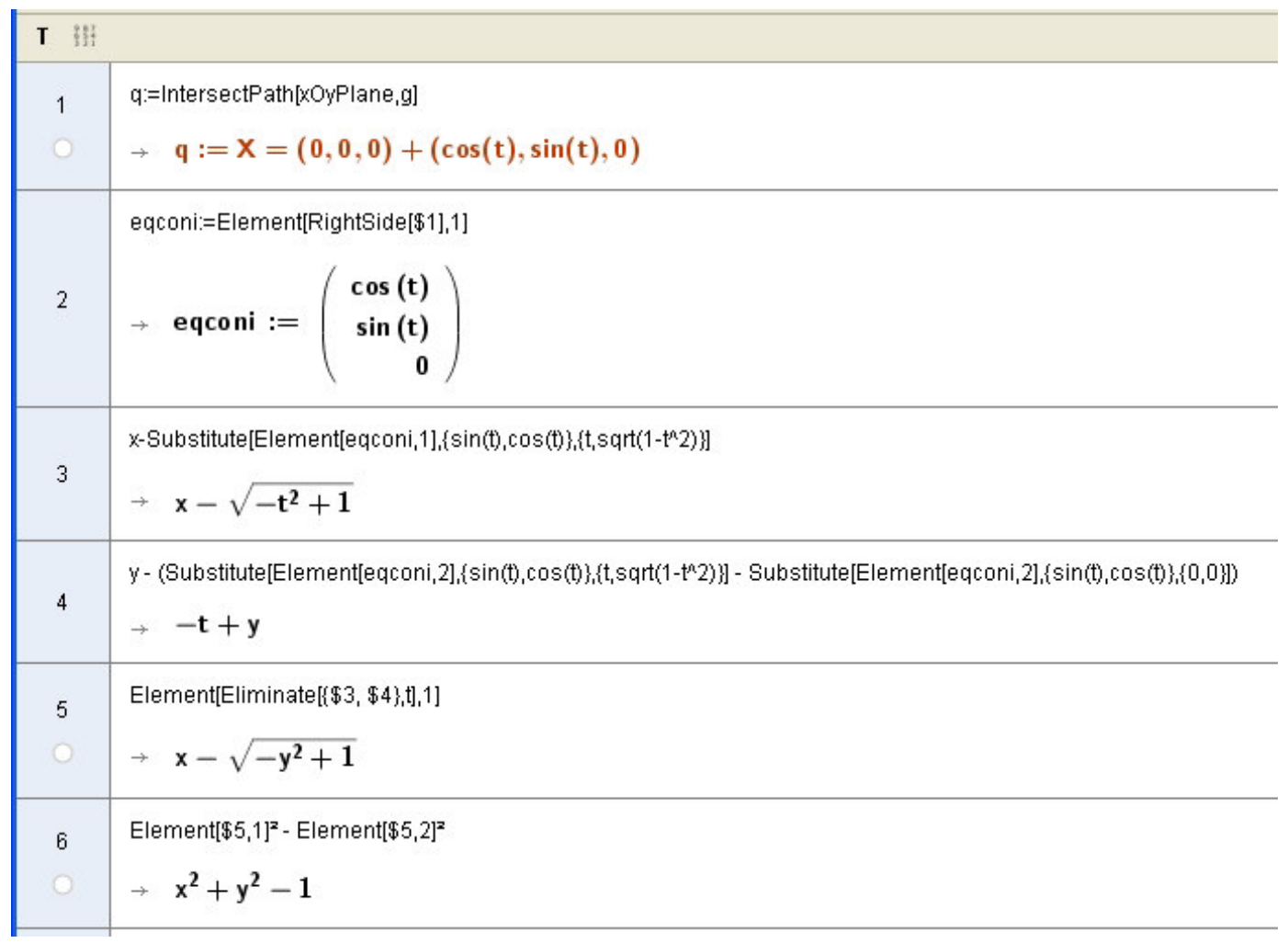

FIGURE 6. Obtaining the implicit equation of a certain circle as a conic section of a right circular cone. 


\begin{tabular}{|c|c|}
\hline \multicolumn{2}{|c|}{ T ilf } \\
\hline 0 & $\rightarrow \mathrm{q}:=\mathrm{X}=(0,0.71,0)+\left(-0.71 \mathrm{t},-0.35 \mathrm{t}^{2}, 0\right)$ \\
\hline 2 & $\begin{array}{l}\text { eqconi:=Element[RightSide[\$1],1] } \\
\rightarrow \text { eqconi }:=\left(\begin{array}{r}-\frac{1136689}{1607521} \mathbf{t} \\
-\frac{1136689}{3215042} \mathbf{t}^{2}+\frac{1136689}{1607521} \\
0\end{array}\right)\end{array}$ \\
\hline 3 & $\begin{array}{l}\left.x \text {-Substitute[Element[eqconi, } 1],\{\sin (t), \cos (t)\},\left\{t, s q r t\left(1-t^{n} 2\right)\right\}\right] \\
\rightarrow \frac{1136689}{1607521} \mathbf{t}+\mathrm{x}\end{array}$ \\
\hline 4 & $\begin{array}{l}\left.\left.\left.\left.y-(\text { Substitute[Element[eqconi, } 2],\{\sin (\mathrm{t}), \cos (\mathrm{t})\},\left\{\mathrm{t}, \mathrm{sqr}\left(1-\mathrm{t}^{n} 2\right)\right\}\right]- \text { Substitute[Element[eqconi, } 2\right],\{\sin (\mathrm{t}), \cos (\mathrm{t}), \mathrm{t}\},\{0,0,0\}\right)\right\} \\
\rightarrow \frac{1136689}{3215042} \mathbf{t}^{2}+\mathbf{y}\end{array}$ \\
\hline 5 & $\begin{array}{l}\text { Element[Eliminate }[\$ 3, \$ 4\}, t], 1] \\
\rightarrow \quad 1607521 x^{2}+2273378 y\end{array}$ \\
\hline 6 & $\begin{array}{l}\$ 5 / \text { abs }(\text { Substitute }[\$ 5,\{x, y\},\{0,1\}]) \\
\rightarrow \frac{1607521}{2273378} \mathrm{x}^{2}+\mathbf{y}\end{array}$ \\
\hline 7 & $\begin{array}{l}\$ 6 \\
\approx 0.71 x^{2}+y\end{array}$ \\
\hline
\end{tabular}

FIGURE 7. Obtaining the implicit equation of a certain parabola as a conic section of a right circular cone.

\section{Conclusions}

We believe that this approach to the conic sections of a right circular cone allows to obtain very simple expressions of the conics as vector equations and of some of them in implicit form. Although obtaining the conic sections using a 3D DGS is done elsewhere, the approach presented here is, as far as we know, new.

The main advantage of this approach with respect to the classic one (cutting the cone through variable planes) is the possibility to easily recognize the curves obtained (compare the equation in the example of Section 1 with the equations obtained in Section 4). This is important from the educational point of view, as the student doesn't have to deal with the equation of a (flat) curve in the 3D space, that has to be classified to be recognized, but with the reduced equation of a conic, what is far simpler and more convenient, as it allows the conic to be directly recognized.

The activity is new and hasn't been proposed to students in the classroom yet. It is planned to be experimented at the end of this academic year. 
A brief note on the approach to the conic sections of a right circular cone from dynamic geometril

\section{References}

[1] E. Algaba-Durán, F. Mayoral-Masa, A. J. Rodríguez-Luis. Apuntes de Álgebra. Ingeniería Industrial, Escuela Técnica Superior de Ingenieros, Universidad de Sevilla, 2009. Available from: http:// www.matematicaaplicada2.es/data/pdf/1260642661_138730054.pdf. Accessed 6 December 2016.

[2] Anonymous. GeoGebra 5.0 Manual, 2016. Available from: https://www.geogebra.org/ wiki/en/Manual. Accessed 6 December 2016.

[3] J. Fernández-Biarge. Estudio de las secciones planas de las cuádricas mediante sus invariantes métricos. Bol. Soc. Puig Adam 63 (2003) 32-44.

[4] F. Botana, A parametric approach to 3D dynamic geometry, Mat. Comp. Simul. 104 (2014) 3-20. DOI: 10.1016/j.matcom.2012.12.004

[5] F. Botana, M. A. Abánades, J. Escribano, Exact internet accessible computation of paths of points in planar linkages and diagrams, Comp. Appl. Eng. Educ. 19/4 (2011), 835-841. DOI: 10.1002/cae.20346

[6] E. Roanes-Lozano, A constructive approach to the quadrics of revolution using 3D dynamic geometry systems with algebraic capabilities, Comput. Appl. Eng. Educ. (To Appear). DOI: 10.1002/cae.21775.

[7] E. Roanes-Lozano, N. van Labeke, E. Roanes-Macías, Connecting the 3D DGS Calques3D with the CAS Maple, Math. Comp. Simul. 80/6 (2010), 1153-1176. DOI: 10.1016/j.matcom.2009.09.008

[8] E. Roanes-Macías, E. Roanes-Lozano, A Maple Package for Automatic Theorem Proving and Discovery in 3D-Geometry, in F. Botana and T. Recio (eds.) Automated Deduction in Geometry, 6th International Workshop, ADG 2006. Springer-Verlag, Lecture Notes in Artificial Intelligence 4689, 2007, pp. 171-188. DOI: 10.1007/978-3-540-77356-6_11

[9] G. S. Stoudt, Can You Really Derive Conic Formulae from a Cone? - Conics as Orthogonal Sections of Cones. Available from: http://www.maa.org/press/periodicals/convergence/ can-you-really-derive-conic-formulae-from-a-cone-conics-asorthogonal-sections-of-cones-introduction. Accesed 6 December 2016.

[10] http: / /www. calques3d. org/. Accessed 6 December 2016.

[11] http://www.cabri.com/cabri-3d.html. Accessed 6 December 2016

\section{Acknowledgments}

This work was partially supported by the research projects TIN2015-66471-P (Government of Spain) and CASI-CAM S2013/ICE-2845 (Comunidad Autónoma de Madrid) and the Research Group ACEIA.

We would also like to thank the anonymous referees for their most valuable comments and suggestions, that helped to improve and clarify the paper.

Eugenio Roanes-Lozano

Instituto de Matemática Interdisciplinar (IMI)

Departamento de Álgebra

Facultad de Educación

Universidad Complutense de Madrid

c/ Rector Royo Villanova s/n

28040-Madrid

Spain

e-mail: eroanesemat.ucm.es 